\title{
An epidemiological study to calculate angle between transepicondylar axis and posterior condylar axis of distal femur on MRI in Indian population
}

\author{
Uikey S. ${ }^{1}$, Mehta S. ${ }^{2}$, Agrawal R. ${ }^{3}$, Goyal N. ${ }^{4}$ \\ ${ }^{1}$ Dr. Suresh Uikey, Assistant Professor, ${ }^{2}$ Dr. Sonu Mehta, Senior Resident, Gandhi Medical College Bhopal, ${ }^{3}$ Dr. Rahul \\ Agrawal, Registrar, Fortis Hospital, Mulund, ${ }^{4}$ Dr. Nikita Goyal, Lecturer, Sri Aurobindo Institute of Medical Science, Indore, \\ MP, India.
}

Corresponding Author: Dr. Sonu Mehta, Senior Resident, Gandhi Medical College Bhopal, Email: sonu_mehta1987@yahoo.com

\begin{abstract}
Introduction: Rotational positioning of femoral component is a critical aspect of total knee arthroplasty and evaluation of the landmark for rotation of distal femur is a challenge. Most Orthopaedic Surgeons prefer to use more available posterior femoral condyle axis. Objectives: Aim of this study is to calculate the angle between TEA and PCA of distal femur on MRI in Indian population. This study is focused on the fact that angle between TEA and PCA may vary from 3 degrees and not fixed as most of the surgeons consider and most of the TKA implants are designed considering it fixed 3 degrees which can seriously affect the outcome and longevity of TKA. Method: In this study MRI KNEE of 152 patients were studied to calculate angle between TEA AND PCA of distal femur. Software called LEONARDO wasutilized to calculate the angle on MRI images. Results: Study shows the average angle of 4.54 degrees. No gender related disparity noted. No age-related increase or decrease of angle noted. In our study we found that, minimum angle between TEA and PCA is 2 degrees while maximum angle is 7.1 degrees. Conclusion: Based on our study and results we conclude that the average angle between TEA and PCA in Indian population is 4.54 degrees. Thus, further studies will be required to investigate the effect of this angle over rotational alignment of femoral component during TKA's and its immediate and longtermoutcomes. Study proves the angle not fixed to 3 degrees and individualistic approach from case to case basis maybe beneficial.
\end{abstract}

Key words: Epidemiological, arthroplasty, TEA.

\section{Introduction}

Rotational positioning of femoral component is a critical aspect of total knee arthroplasty and evaluation of the landmark for rotation of distal femur is a challenge. Most Orthopaedic Surgeons prefer to use more available posterior femoral condyle axis, but other landmark especially surgical transepicondylar axis may be more valid. MRI scan helps to evaluate these landmarks more accurately. The purpose of our study was to ascertain the angle between transepicondylar axis and posterior condylar axis in Indian population on MRI scan. Most of the surgeons consider it as fixed 3 degrees of external rotation in all the cases but purpose of this study is to actually calculate it on MRI images of Indian population. As rotational alignment plays an immense role in TKA outcomes it is important to put the implant in geometrically anatomical position.

The geometry of the proximal tibia and distal femur is intimately linked with the biomechanics of the tibio-femoral and patella-femoral joints [1]. Rotational positioning of femoral and tibial component is appeared as critical aspect of total knee arthroplasty (TKA). Since the consequences of mal rotation may produce patellofemoral problems, flexion instability, stiffness, and abnormal gait patterns [2,3]. Despite the current high success rate of TKA, patellofemoral complication remains one of common cause of revision surgeries[3]. Proper positioning of TKA components is vital for the functional outcome of the procedure. Although the desired positions and guiding landmarks for placement of the femoral and tibial components in the coronal and sagittal planes have been well defined, rotational positioning of the components might still be challenging [4].

Manuscript Received: $28^{\text {th }}$ November 2017

Reviewed: $7^{\text {th }}$ December 2017

Author Corrected: $14^{\text {th }}$ December 2017

Accepted for Publication: $20^{\text {th }}$ December 2017 


\section{Original Research Article}

In the field of total knee arthroplasty, position of the implants in axial plane is referred to as rotational alignment[1]. One of the most common mistakes in TKA is femoral component malrotation, especially in internal rotation. If the femoral component is inserted inappropriately, malrotation induces patellofemoral maltracking and flexion gap mismatch. patellar maltracking eventually results in patellar maltracking eventually results in patella dislocation or severe limited range of motion and flexion gap mismatch causes knee instability. these complications affect the postoperative clinical outcome and patient's satisfaction and reduce the lifespan of prosthesis. Multiple researches have confirmed the relation between malrotation of femoral component and early failure of TKA[5,6].

As mentioned above it is important to know the exact rotational alignment of distal femur prior to surgery. Although there are several reference lines to assess the rotation of distal femur that help to mark the best cut from posterior aspect of femoral condyles, but there is still disagreement about their significant and mutual angular relationship[1,7]. Posterior cut of femur can be done relative to its relation with the tibia[1].Distal femoral references include the posterior femoral condylar axis (PCA) and the transepicondylar axis (TEA). The line connecting the tip of the lateral epicondyle to the medial epicondylar sulcus as defined according to Berger et al[8]. The transepicondylar axis is a valid reference for both the femur and tibia probably because it approximates theflexion.

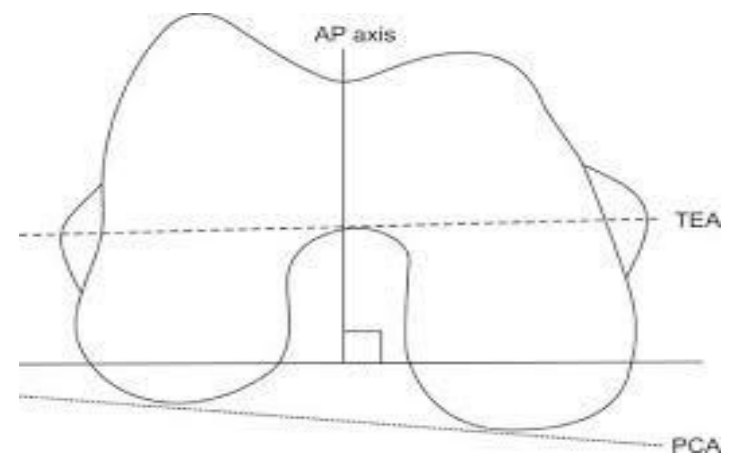

Fig-1: Distal femoral anatomy showing various axes

Significance of Measuring angle between TEA and PCA- Transepicondylar Axis, The TEA is a line connecting the prominence of the lateral epicondyle to the medial epicondylar ridge (clinical TEA) or the medial epicondylar sulcus (surgical TEA)[8,9]. Approximating the flexion-extension axis of the knee, the TEA corresponds to the femoral collateral ligamentous origin ${ }^{9}$. According to Berger et al[8]. The surgical TEA can be a useful landmark in determining the neutral rotational orientation of the femoral component. Enhanced central patellofemoral tracking and improved femorotibial kinematics can be obtained by placing the femoral component parallel to the TEA[9,10]. A lower incidence and magnitude of femoral condylar lift-off (better coronal stability) was demonstrated by Insall et al[10]. If the femoral component was placed parallel to the TEA axis in a kinematic analysis. Placement of the femoral component parallel to the TEA assists in obtaining a rectangular flexion gap (90\% using the TEA, $83 \%$ using the AP axis, and $70 \%$ using the posterior condylar axis) in an analysis performed by Olcott and Scott [9].

The TEA can also be referred in revision TKA and in Primary TKA where there is posterior condylar hypoplasia or erosion. Unfortunately, numerous studies report that surgeons may be unable to accurately and reproducibly identify the TEA. Locating the medial and lateral epicondyles precisely is often difficult to reproduce intra operatively[11]. Jerosch et al. compared the difference in position of epicondyles marked by surgeons under experimental conditions. They demonstrated that the range of position chosen by the surgeons on the medial side varied by $22.3 \mathrm{~mm}$. They found that the range of position on the lateral epicondyle also varied by $13.8 \mathrm{~mm}[11]$. In a series of $74 \mathrm{TKA}$, Kinzel et al studied the accuracy of epicondylar identification. Intraoperatively, they placed pins in the femoral epicondyles. When evaluated with postoperative CT scans, the epicondyles were correctly identified to with in $\pm 3^{\circ}$ in only $75 \%$ of the cases.

They note dawiderangeoferror (six degrees of external rotationto1 1degrees of internal rotational error) and concluded that the TEA was an unreliable landmark to determine femoral component rotation [12]. When the TEA was used to determine femoral component rotation, Yau et al found that $56 \%$ of the time a range of error greater than five degrees occurred. They also noted a wide range of error in intraoperative surgeon identification of the femoral epicondyles (28 degree error range; 11 degrees external rotation to 17 degrees of internal rotation)[13]. In a cadaveric study using an imageless computer navigation 


\section{Original Research Article}

system, Siston et alcompared the use of the posterior condylar, anteroposterior and transepicondylar axes to set femoral component rotation. Only $17 \%$ of the actual registered landmarks fell within five degrees of the true epicondylar axis. Each of the 11 surgeons who participated in the study registered landmarks that tended to overly externally rotate the femoral component relative to the true TEA[14]. Benjaminfound that the TEA was only accurate to within one degree $34 \%$ of the time. The posterior condylar axis most frequently corresponded to the rotation alignment of the implant[15].

\section{Material and Methodology}

In this study 152 patient of age between 14-72 years were evaluated who has undergone MRI knee at a tertiary care hospital for any reason between 01/05/2014 to 31/01/2015. Axial cuts of MRI KNEE were used to calculate angle between TEA and PCA on PACS using software installed at Fortis Hospital,Mulund.

Inclusion Criteria- All skeletally mature Indian people who are willing to give consent for the study and do not have any distortion of distal femoral bony anatomy. These people who would have MRI KNEE done anyway for other cause and therefore are not being subjected to this investigation as an additional expense purely for the purpose of this study.

\section{Exclusion Criteria}

- Patients had distal femoral fractures distorting distal femoralanatomy.

- Patients having distorted bony anatomy due to some other medicalillness.

\section{Method of Measurement[16]}

Magnetic resonance imaging (MRI) is becoming widely used in orthopaedic surgery.

It has unique advantages over other imaging modalities. First, it is totally non-invasive and has no known adverse effects on the human body. Secondly, it can provide information not only on the density of tissues but also on their chemical structure. Thirdly, MRI can provide a direct three-dimensional view of the part examined. Until now the knee has not been explored in detail. For the diagnosis of knee disorders, an understanding of the normal knee is clearly essential and the aim of this present study was to learn the distal femoral anatomy on MRI appearance of the knee.

MRI was performed using a 1.5 Tesla whole body MR imaging system with an extremity coil, and all the subjects were restrained from moving during the scanning process. Pulse sequences were T2-weighted images. The direction of axial slice imaging placed the slice perpendicular to the femoral mechanical axis in the coronal plane and perpendicular to the long axis of the femur in the sagital plane. The axial slice on the most prominent part of both femoral condyles was selected for analysis. The transepicondylar axis was defined as a line between the most medial and the most lateral prominences of the epicondyles(clinical TEA) or most prominent point of lateral epicondyle to medial epicondylar sulcus (surgical TEA), and the posterior condylar tangent as a line connecting the posterior aspects of the femoral condyles. Articular boundaries of the femoral condyles were used for posterior condylar tangent. The surgical TEA was considered as reference axis while calculating the angle between TEA and PCA in all subjects to maintain uniformity in all patients and to reduce the error.

Angle between these two lines were calculated and documented.



Fig-2: Axial cut of distal femur on MRI showing measurement of angle between TEA And PCA 



Fig-3: Distal Femur Anatomy Showing Relationship Between TEA And PCA

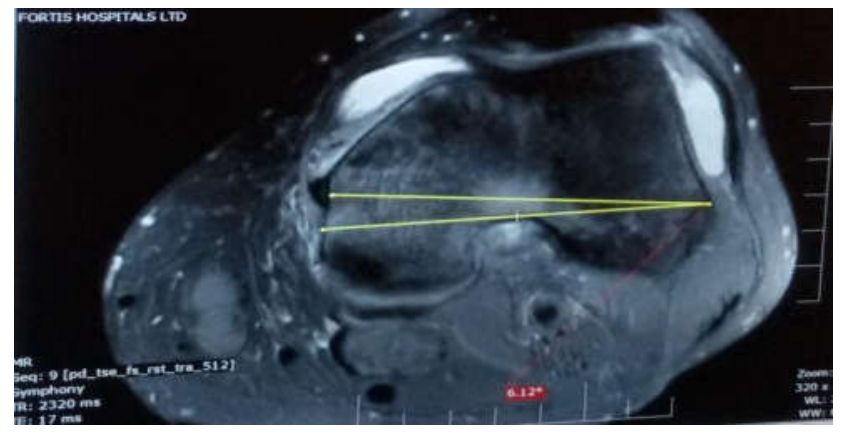

Fig-4: Axial MRI Slice Showing Measurement of Angle Between TEA And PCA

\section{Results}

Age Related Distribution- In this study 152 patients were evaluated for their MRI KNEE. Out of 152 patients 22 patients were under 20 years of age.36 patients were in 21-30 years of age.35 patients were in 31-40 years of age. 22 patients were in 41-50 years of age. 27 patients were in 51-60 years of age. 10 patients were above 60 years of age. Youngest patient was 14 years and oldest patient was 72 years old.

Table No.-1:Age Distribution.

\begin{tabular}{|c|c|c|c|c|}
\hline & Mean & SD & Min & Max \\
\hline Age & 37.58 & 15.07 & 14.0 & 72.0 \\
\hline Angle between TEA and PCA & 4.54 & 1.14 & 2.00 & 7.10 \\
\hline
\end{tabular}

Mean Values of Age and Angle as per study.

Gender Distribution- Out of 152 patients 62 were male and 90 were female. thus showing male preponderance.

Sidewise Distribution-In our study out of 152 patients left side affected patients were 71 whereas right side affected were 81 . Distribution According To Indication Of MRI- Most common cause to undergo MRI KNEE was knee pain (57 patients). other causes were ACL tear (22 patients), instability (19 patients),knee injury(41 patients), tibia platue fracture (13 patients).

Table No.-2: Reasons to undergo MRI.

\begin{tabular}{|c|c|c|}
\hline Reason to undergo MRI & Count & Percentage \\
\hline Clinically ACL tear & 22 & 14.5 \\
\hline Instability & 19 & 12.5 \\
\hline Knee Injury & 41 & 27.0 \\
\hline Knee Pain & 57 & 37.5 \\
\hline Tibia Platue Fracture & 13 & 8.6 \\
\hline Total & 152 & 100.0 \\
\hline
\end{tabular}

Most common cause for undergoing MRI was knee pain. 
Original Research Article

Table No.- 3: Analysis according to reason to undergo MRI.

\begin{tabular}{|c|c|c|c|c|}
\hline \multirow[t]{2}{*}{ Reason to undergo MRI } & \multicolumn{3}{|c|}{ Angle between TEA and PCA } & \multirow[b]{2}{*}{ Max } \\
\hline & Average & SD & Min & \\
\hline Clinically ACL tear & 4.72 & 1.27 & 2.15 & 7.00 \\
\hline Instability & 4.41 & 1.01 & 3.00 & 6.11 \\
\hline Knee Injury & 4.35 & 1.17 & 2.00 & 7.10 \\
\hline Knee Pain & 4.55 & 1.13 & 2.50 & 7.00 \\
\hline Tibia Platue Fracture & 4.91 & 1.06 & 3.45 & 6.55 \\
\hline
\end{tabular}

Etiology of Pain was incidental and mri used to calculate anatomical angles irrespective of etiology of pain.

Table No.-4: Analysis according to age groups.

\begin{tabular}{|c|c|c|c|c|}
\hline Age Group & \multicolumn{2}{|c|}{ Angle between TEA and PCA } & Min & Max \\
\hline & Average & SD & 3.00 & 7.10 \\
\hline <= 20 yrs & 4.76 & 1.15 & 2.90 & 6.60 \\
\hline $\mathbf{2 1 - 3 0}$ yrs & 4.82 & 1.02 & 2.00 & 7.00 \\
\hline $\mathbf{3 1 - 4 0}$ yrs & 4.41 & 1.08 & 2.90 & 6.58 \\
\hline $\mathbf{4 1 - 5 0}$ yrs & 4.62 & 1.23 & 2.50 & 7.00 \\
\hline $\mathbf{5 1 - 6 0}$ yrs & 4.21 & 1.21 & 2.65 & 5.90 \\
\hline$>\mathbf{6 0}$ yrs & 4.13 & 1.20 & & \\
\hline
\end{tabular}

No age correlation can be ascertained.

Table No.-5 Analysis according to gender.

\begin{tabular}{|c|c|c|c|c|}
\hline Gender & \multicolumn{2}{|c|}{ Angle between TEA and PCA } & Min & Max \\
\hline & Average & SD & 2.15 & 7.00 \\
\hline Female & 4.63 & 1.13 & 2.00 & 7.10 \\
\hline
\end{tabular}

No gender correlation could be ascertained.

Using One way ANOVA, our p values is $0.395(>0.05)$ therefore there is significant difference between angles. Normally taken angle is 3 degrees but the study indicates average angle 4.54 degrees. The difference being 1.54 degrees is statistically significant.

\section{Discussion}

Proper rotational alignment of the component is one of the most important factors for successful total knee arthroplasty[17]. Previous studies recommended that the femoral component should be inserted parallel to the transepicondylaraxis[18,19]. However, accurate detection of both the medial and lateral epicondyle is sometimes difficult $[18,19,20]$, as is finding the AP axis, because of trochlear wear or intercondylar osteophytes in arthritic knees[21]. The posterior condyles can be more easily identified intraoperatively, therefore some bone cutting guide systems are designed to align the femoral component in 3-5" of external rotation from the posterior condyles. These systems align the femoral component parallel to the transepicondylar axis with a small amount of deviation, because the previous anatomical studies have shown that the transepicondylar axis is externally rotated from the posterior condylar tangent in 3-6". Therefore, there is need of studies which will give the preoperative idea of distal femoral anatomy and transepicondoylar axis to posterior condylar axis angle so as to develop preoperative plan of femoral component placement.

The goal of this study is to calculate angle between TEA and PCA on MRI knee. An important part of this work was dedicated to the development of a model based on a MRI Images for study of knee anatomy. The human knee has an impressive potential for adapting its kinematics depending on the conditions. In that respect, it is interesting to calculate angle between TEA and PCA in Indian population in order to study various reasons for knee pain in normal knees and even after total knee arthroplasty. Total Knee Arthroplasty is a permanent 


\section{Original Research Article}

method to relieve pain in knee due to various reasons. It is of utmost important to put TKA implants in alignment as close as the normal knee anatomy. To put implants in normal anatomical position one has to know about the relationship between various axes of knee joint.

The strength of this study is that all the MRI SCANS were done with uniform technique on single scanner with same amount of slice thickness. The angles were calculated on the axial cut image. the most prominent point of the epicondyle was considered to be the reference point in order to minimiseerrors.

The limitations of this study is sample size is less and the inconspicuous morphology of medial epicondyle. which leads to high margins of error which will affect rotational alignment of knee joint. The methodology allows to detect and describe the angle between TEA and PCA in different age groups, Also the reason for undergoing MRI knee was different.

In the study of Aglietti P et al. they evaluated the angular difference between different alignment axes and the effect of TEA AND PCA alignment axes on implant placement and amount of bone resected on seven implant systems. There study is based on computed tomography scan[2]. The TEA is a valid reference for boththe femur and tibia probably because it approximates the flexion-extension axis of the knee and the femoral collateral ligaments origin. Their data confirm this concept. In the femur, rotational alignment is based on coronal deformity. In the tibia, they observed high interindividual variability and no relationship either to gender or deformity[2].

In the study of gujharathi et al. anterior and posterior referencing systems are used to obtain correct component alignment while avoiding notching of the anterior cortex of the femur. In their study they have showed that periprosthetic supracondylar fracture has been reported to range from $0.3 \%$ to $4.8 \%$ after TKA due to mal-alignment of the femoral component[22].

In study of Mont MA et al they used intramedullary goniometer intraoperative to improve malalignment. they found that Inaccuracies in component sizing and placement can be strongly related to outcome of TKAs. There studies have shown anterior notching to be present in $40 \%$ to $52 \%$ of reported fractures[23].

Our study has focused mainly on femoral prosthesis malalignment with respect to the different axes of the distal femur. There is no agreement on how to best account for morphological variation during cutting techniques and component placement. Hence, the purpose of the study is to come up with a reproducible algorithm to simulate what the surgeon does in the operating room. This method could help in surgical preplanning, patient specific instruments, and implant design.

Berger et $\mathrm{Al}$ in their study of determination of rotational alignment of femoral component in primary total knee arthroplasty using transepicondylar axis they have shown that Measurement of the posterior condylar angle referenced from the surgical epicondylar axis yielded a mean posterior condylar angle of 3.5 degrees $(+/-1.2$ degrees) for males and a mean posterior condylar angle of 0.3 degree (+/- 1.2 degrees) for females[8].

This signifies the need of further study on the anatomy of distal femoral axes in Indian population. our study has evaluated the gender difference in the anatomy of distal femur and documented that differently.

In our study we found the main reason for undergoing MRI knee was knee injury excluding distal femur fractures. the mean angle we measured was 4.54 degrees which is in accordance with the study done by Berger et al.

\section{Conclusion}

Based on our study and results we conclude that the average angle between TEA and PCA in Indian population is 4.54 degrees. Thus further studies will be required to investigate the effect of this angle over rotational alignment of femoral component during TKA's and its immediate and long term outcomes.

Conflict of interest: None declared. Funding: Nil, Permission from IRB: Yes

\section{Bibliography}

1. Victor J.Rotational alignment of the distal femur: A literature review. OrthopTraumatol BurgErs.2009;95(5): 365-372.

2. AgliettiP,sensiL,Cuomo,Ciardullo.Rotational position of femoral and tibial component in TKA using the femoral transepicondylaraxis .clinOrthopRelat Res.2008; 466 (11):2751-275.

3. Rossi R, Bruzzone, Bonasia, MarmottA, Castoldi. Evaluation of tibial rotational alignment in total knee arthroplasty:a cadaver study.KneeSurg Sports Traumatol Arthrosc. 2010;18(7)889-893.

4. Zhang XL, Zhang W, Shao JJ. Rotational alignment in total knee arthroplasty; non image based navigation 


\section{Original Research Article}

system versus conventional technique.Chin Med J.(Engl). 2012; 25(2):236-243.

5. SunT, LvH, Hong. Rotational landmarks and total knee arthroplasty in osteoarthritis knees ZhongguoXiu fu.2007; 21(3): 226-230.

6. UeharaK, KadoyaY, KobayashiA, OhashiH,YamanoY. Boneanatomy and rotatonal alignment total knee arthroplasty. Clin Orthop RelatRes. 2002 ;402:196-201.

7. Berhouet J, Beaufils P, Boisrenoult P, FrascaD, PujolN. Rotatinal positioning of the tibial tray in total knee arthroplasty; a CT evaluation OrthopRelat Res. 2011;97 (7): 699-704.

8. Berger RA, Rubash HE, Seel MJ, Thompson WH, Crossett LS. Determining the rotational alignment of femoral component in total knee arthroplasty using epicondylar axis,.ClinOrthopRelat Res.1993;286:40-47.

9. Olcott CW, Scott RD. A comparison of 4 intraoperative methods to determine femoral component rotation during total knee arthroplasty. J Arthroplasty.2000;15(1):22-6.

10. Insall JN, Scuderi GR, Komistek RD, Math K, Dennis DA, Anderson DT. Correlation between condylar lift-off and femoral component alignment. ClinOrthopRelat Res. 2002;(403):143-52 11. J.

11. Jerosch J, Peuker E, Philipps B, Filler $T$. Interindividual re- producibility in perioperative rotational alignment of femo- ral components in knee prosthetic surgery using the tran- sepicondylar axis. Knee Surg Sports TraumatolArthrosc. 2002;10(3):194-7.

12. Kinzel V, Ledger M, Shakespeare D. Can the epicondylar axis be defined accurately in total knee arthroplasty? Knee.2005;12(4):293-6.

13. Yau WP, Chiu KY, Tang WM. How precise is the determination of rotational alignment of the femoral prosthesis in total knee arthroplasty: an in vivo study. $\mathrm{J}$ Arthroplasty.2007;22(7):1042-8.
14. Siston RA, Patel JJ, Goodman SB, Delp SL, Giori NJ. The variability of femoral rotational alignment in total knee ar- throplasty. J Bone Joint Surg Am. 2005;87 (10): 2276-80.

15. Benjamin J. Determining femoral component position us- ing CAS and measured resection. Clin Orthop Relat Res. 2008;466(11):2745-50.

16. Alifidi RJ, Haaga JR. El Yousef SJ, et a!. Preliminary experimental results in humans and animals with a superconducting, wholebody. nuclear magnetic resonance scanner. Radiology 1982; 143: 17581.

17. Akagi M, Matsusue $Y$, Mata $T$, et al. Effect of rotational alignment on patellar tracking in total knee arthroplasty. ClinOrthop1999;366:155-63,

18. Berger RA, Crossett LS, Jacobs JJ, Rubash HE. Malrotation causing patellofemoral complications after total knee arthroplasty. ClinOrthop1998;356:14\&53.

19. Griffin FM. Insall JN, Scuderi GR. The posterior condylar angle in osteoarthritic knees. J Arthroplasty 1988;12:812-5.

20. Katz MA, Beck TD, Silber JS, et al. Determining femoral rotational alignment in total knee arthroplasty: reliability of techniques. J Arthroplasty2001;16:30145.

21. Poilvache PL, Insall JN, Scuderi GR, Font-Rodriguez DE. Rotational landmarks and sizing of the distal femur in total knee arthroplasty. ClinOrthop1996;331:3546.

22. Gujarathi N, Putti AB, Abboud RJ, MacLean JG, Espley AJ, Kellett CF. Risk of periprosthetic fracture after anterior femoral notching. Acta Orthop. 2009; 80: $553-556$.

23. Mont MA, Urquhart MA, Hungerford DS, Krackow KA. Intra- medullary goniometer can improve alignment in knee arthroplasty surgery. J Arthroplasty. 1997;12: 332-336

\section{How to cite this article?}

Uikey S, Mehta S, Agrawal R, Goyal N. An epidemiological study to calculate angle between transepicondylar axis and posterior condylar axis of distal femur on MRI in Indian population. Surgical Update: Int J surg Orthopedics.2017;3(4):175181.doi:10.17511/ijoso.2017.i04.14. 